\title{
Estimation of nuclear matrix elements of double- $\beta$ decay from shell model and quasiparticle random-phase approximation
}

\author{
J. Terasaki ${ }^{1, \mathrm{a}} \mathbb{D}$, Y. Iwata $^{2}$ \\ ${ }^{1}$ Institute of Experimental and Applied Physics, Czech Technical University in Prague, Husova 240/5, \\ 11000 Prague 1, Czech Republic \\ 2 Faculty of Chemistry, Materials and Bioengineering, Kansai University, Yamatemachi 3-3-35, Suita, \\ Osaka 564-8680, Japan
}

Received: 16 April 2021 / Accepted: 19 August 2021

(C) The Author(s) 2021

\begin{abstract}
The nuclear matrix element (NME) of neutrinoless double- $\beta(0 \nu \beta \beta)$ decay is an essential input for determining the neutrino effective mass, if the half-life of this decay is measured. Reliable calculation of this NME has been a long-standing problem because of the diversity of the predicted values of the NME, which depends on the calculation method. In this study, we focus on the shell model and the QRPA. The shell model has a rich amount of the many-particle many-hole correlations, and the quasiparticle random-phase approximation (QRPA) can obtain the convergence of the calculation results with respect to the extension of the single-particle space. It is difficult for the shell model to obtain the convergence of the $0 \nu \beta \beta$ NME with respect to the valence single-particle space. The many-body correlations of the QRPA may be insufficient, depending on the nuclei. We propose a new method to phenomenologically modify the results of the shell model and the QRPA compensating for the insufficiencies of each method using the information of other methods in a complementary manner. Extrapolations of the components of the $0 v \beta \beta$ NME of the shell model are made toward a very large valence single-particle space. We introduce a modification factor to the components of the $0 v \beta \beta$ NME of the QRPA. Our modification method yields similar values of the $0 \nu \beta \beta$ NME for the two methods with respect to ${ }^{48} \mathrm{Ca}$. The NME of the two-neutrino double- $\beta$ decay is also modified in a similar but simpler manner, and the consistency of the two methods is improved.
\end{abstract}

\section{Introduction}

Neutrinoless double- $\beta(0 \nu \beta \beta)$ decay has been studied intensively by many researchers since the prediction by Ref. [1] as one of the clues for the new physics. Finding this decay implies that the neutrino is a Majorana particle, and the lepton number is not conserved. In addition, if the half-life is measured, it is possible to determine the effective neutrino mass, which is also called the Majorana mass. The neutrino was thought to be a particle that was either massless or very light for many decades until the discovery of the neutrino oscillation [2-5],

\footnotetext{
a e-mail: jun.terasaki@cvut.cz (corresponding author)
} 
which has proven its massiveness. Currently, determination of the mass scale of neutrino is one of the major subjects of neutrino physics.

The $0 \nu \beta \beta$ decay can be used as one of the limited methods for determining this mass scale. Approximately 20 large-scale experimental projects [6-23] are in progress to observe the $0 \nu \beta \beta$ decay with the assumption of the Majorana nature of the neutrino. For other methods of using nuclei, see Refs. [24-26]. With the current status, i.e., no successful report of observing the $0 \nu \beta \beta$ decay, the upper limit of the effective neutrino mass is deduced, for example, in Ref. [16], from the performance of the detector system, the theoretically calculated nuclear matrix element (NME), and the phase-space factor originating from the emitted electrons. The NME is more difficult to calculate accurately than the phase-space factor because nuclear wave functions are necessary. The lightest nucleus used for the experiments is ${ }^{48} \mathrm{Ca}$ [6], and some approximation is essential for the wave functions of the involved nuclei; this necessity is obvious for heavier candidate nuclei.

It is well known that the calculated $0 \nu \beta \beta$ NMEs are distributed in the range of a factor of 2 to 3, depending on the theoretical method used to calculate the nuclear wave functions $[27,28]$. In particular, the shell model and quasiparticle random-phase approximation (QRPA) ${ }^{1}$, which have been used intensively and historically, have a difference of a factor of two for several instances of the $0 \nu \beta \beta$ decay [27,28]. The shell model is the diagonalization method of the many-body Hamiltonian, and many-particle many-hole (mpmh) correlations are included in the wave functions. We define mpmh as at least two-particle two-hole (2p2h) configuration mixing. The QRPA is an approximation to obtain the transitions from the ground state to excited states, and this transition is limited to two-quasiparticle creation and annihilation. The shell model calculations are performed in many current cases with one major valence shell defined by the harmonic oscillator for the single particles, while the QRPA can use a much larger single-particle space with a more realistic single-particle basis. The limit of the valence single-particle space or many-body correlations is primarily due to the technical limits of computation. The correct $0 \nu \beta \beta$ NME should be confirmed by the convergence of the result with respect to the extension of the valence single-particle space and the mpmh components of the nuclear wave function. Despite the remarkable development of modern computers, it is difficult to achieve this double convergence. The physical origin of this difficulty is the neutrino potential included in the $0 \nu \beta \beta$ NME. This two-body potential has a singularity at the origin; therefore, a very large wave function space is necessary. For the shell model and similar calculations of the $0 \nu \beta \beta$ NME for ${ }^{48} \mathrm{Ca}$, the two-major valence shell is the largest valence single-particle space ever used [30,31]. For the QRPA, an extension called the renormalized QRPA [32,33] has been investigated.

Another problem is the effective axial-vector current coupling, denoted $g_{A}$, for the nuclei. The $0 v \beta \beta$ NME is a linear combination of the Gamow-Teller (GT), Fermi, and tensor components, and the coefficient includes $g_{A}$. The tensor component is omitted in this study because its contribution is small, e.g., [30,34]. The $g_{A}$ is equal to one in the quark-lepton level, e.g., [35], while it is $1.27641(45)_{\text {stat }}(33)_{\text {sys }}$ [36] for the neutron. This difference indicates that $g_{A}$ is affected by the many-body effects of the quarks. Thus, the corrections of the transition operator as a result of the many-body effects of the nucleons may be necessary, if exact nuclear wave functions are available. Approximation of the wave functions causes another necessity for an effective $g_{A}$. There is a long history of theoretically deriving the effective $g_{A}$, e.g., [37]. The method to determine the effective $g_{A}$ has not yet been established for the

\footnotetext{
${ }^{1}$ Most studies with this approach use the proton-neutron QRPA, e.g., [29], which is called the QRPA for simplicity in this paper.
} 
$0 \nu \beta \beta$ decay. For recent attempts to theoretically derive the effective transition operators of this decay, see Refs. [38,39] and references therein.

In this article, we propose a new approach to estimate the components of the $0 \nu \beta \beta$ NME by modifying the results of the calculations of the shell model and the QRPA by compensating for the insufficient points of the two methods. It is difficult for the shell model to obtain the convergence of the $0 \nu \beta \beta \mathrm{NME}$ with respect to the extension of the valence single-particle space. The many-body correlations of the QRPA may be insufficient, depending on the nuclei. Extrapolations of the components of the $0 \nu \beta \beta$ NME of the shell model are made with respect to the energy representing the size of the valence single-particle space, referring to the intermediate-state energy dependence of the components of the $0 \nu \beta \beta$ NME of the QRPA. In addition, we introduce a modification factor to the components of the $0 v \beta \beta$ NME of the QRPA by comparing the charge-change strength functions of the experiments, the shell model, and the QRPA. This modification factor represents the mpmh effects missing in the QRPA. ${ }^{48} \mathrm{Ca}$ is used in this study because the shell model calculations were performed with the one- and two-major valence single-particle spaces [30]. We discuss the GT and Fermi components separately to avoid the involvement of the effective- $g_{A}$ problem.

This paper is organized as follows: in Sect. 2, the basic equations used in this study are summarized. In Sect. 3, we discuss the modification of the GT component of the $0 \nu \beta \beta$ NME of the shell model. Subsequently, the modification of that component of the QRPA is discussed in Sect. 4. Section 5 discusses the Fermi component of the $0 \nu \beta \beta$ NME. The $0 \nu \beta \beta$ NME is calculated from the two components in Sect. 6. In Sect. 7, we discuss the modification of the NME of the two-neutrino double- $\beta(2 v \beta \beta)$ decay. Section 8 provides a summary of the study.

\section{Basic equations}

Prior to subsequent discussion, we summarize the basic equations and definitions of the quantities relevant to the $0 \nu \beta \beta$ decay. The probability of this decay, e.g., [40,41], can be written as

$$
P_{0 v}=\left|M^{(0 v)}\right|^{2} G_{0 v}\left(\frac{\left\langle m_{v}\right\rangle}{m_{e}}\right)^{2},
$$

where $M^{(0 v)}$ is the NME of the $0 \nu \beta \beta$ decay, and $G_{0 v}$ denotes the phase-space factor [42]. The effective neutrino mass is denoted by $\left\langle m_{v}\right\rangle$, and $m_{e}$ is the electron mass. The half-life is inversely proportional to $P_{0 v}$. The $M^{(0 v)}$ discussed in this study is calculated as

$$
M^{(0 v)}=M_{\mathrm{GT}}^{(0 v)}-\left(\frac{g_{V}}{g_{A}}\right)^{2} M_{\mathrm{F}}^{(0 v)} .
$$

$M_{\mathrm{GT}}^{(0 v)}$ and $M_{\mathrm{F}}^{(0 v)}$ denote the GT and the Fermi components, respectively. The constant $g_{V}$ is the vector-current coupling. The GT component with closure approximation, e.g., [43,44], is given by

$$
M_{\mathrm{GT}}^{(0 v)}=\sum_{p n p^{\prime} n^{\prime}}\left\langle p p^{\prime}\left|V_{\mathrm{GT}}^{(0 v)}\left(r ; \bar{E}_{B}\right)\right| n n^{\prime}\right\rangle\left\langle F\left|c_{p}^{\dagger} c_{n} c_{p^{\prime}}^{\dagger} c_{n^{\prime}}\right| I\right\rangle,
$$

where $p$ and $p^{\prime}$ ( $n$ and $n^{\prime}$ ) denote the proton (neutron) states; $c_{i}^{\dagger}$ is the creation operator of the single-particle state $i$ ( $p$ or $n)$; and the annihilation operator is $c_{i} . V_{\mathrm{GT}}^{(0 v)}\left(r ; \bar{E}_{B}\right)$ is the double 
GT transition operator of the $0 \nu \beta \beta$ decay with the two-nucleon distance $r$, and $\bar{E}_{B}$ is the average energy of the intermediate states $|B\rangle$, which are the virtual states between the first and second $\beta$ decays. $|I\rangle$ and $|F\rangle$ denote the initial and final states of the decay, respectively, and the ground states are used. $V_{\mathrm{GT}}^{(0 v)}\left(r ; \bar{E}_{B}\right)$ is defined as

$$
V_{\mathrm{GT}}^{(0 v)}\left(r ; \bar{E}_{B}\right)=h_{+}\left(r, \bar{E}_{B}\right) \sigma(1) \cdot \sigma(2) \tau^{-}(1) \tau^{-}(2),
$$

where $\sigma$ is the spin Pauli operator, and $\tau^{-}$indicates that the operator changes the neutron to the proton. Arguments 1 and 2 distinguish the two particles operated. The function $h_{+}\left(r, \bar{E}_{B}\right)$ is the neutrino potential, whose behavior is similar to that of the Coulomb potential with a singularity at $r=0$. For the equation of $h_{+}\left(r, \bar{E}_{B}\right)$, see Ref. [28,45]. Equation (3) is used by the shell model.

The QRPA uses two sets of intermediate states because of the features of the approximation. One is the $\left|B_{I}\right\rangle$ obtained on the basis of $|I\rangle$, and the other is the $\left|B_{F}\right\rangle$ based on $|F\rangle$. The equation used for $M^{(0 v)}$ in the QRPA approach reads

$$
M_{\mathrm{GT}}^{(0 v)}(\mathrm{QRPA})=\sum_{B_{F} B_{I}} \sum_{p n p^{\prime} n^{\prime}}\left\langle p p^{\prime}\left|V_{\mathrm{GT}}^{(0 v)}\left(r ; \bar{E}_{B}\right)\right| n n^{\prime}\right\rangle\left\langle F\left|c_{p}^{\dagger} c_{n}\right| B_{F}\right\rangle\left\langle B_{F} \mid B_{I}\right\rangle\left\langle B_{I}\left|c_{p^{\prime}}^{\dagger} c_{n^{\prime}}\right| I\right\rangle .
$$

Below, we refer to this as $M_{\mathrm{GT}}^{(0 v)}$ of the QRPA. The intermediate states are explicitly used because the QRPA is suitable for calculating the transition density matrix elements $\left\langle F\left|c_{p}^{\dagger} c_{n}\right| B_{F}\right\rangle$ and $\left\langle B_{I}\left|c_{p^{\prime}}^{\dagger} c_{n^{\prime}}\right| I\right\rangle$. For calculation of the overlap $\left\langle B_{F} \mid B_{I}\right\rangle$, see Refs. [46,47]. The equations for the Fermi component are the same as those in Eqs. (3)-(5), except that the double-spin operator $\sigma(1) \cdot \sigma(2)$ is not used.

\section{Modification of GT component of $0 v \beta \beta$ NME of shell model}

\subsection{Method with the help of experimental strength function}

Our study is based on the shell model results of Ref. [30] and the QRPA results of Ref. [48] for two reasons: one is that Ref. [30] includes one- and two-major valence shell calculations. The extension of the valence single-particle space is important in our study. The other reason is that the energy dependences of the charge-change strength functions of the two methods are similar in the energy region up to the GT giant resonance (see Refs. [30,48]). Thus, the physical effects of the interactions used in the two calculations are similar. This similarity is expected because both interactions are phenomenological. The shell model results used in this study were obtained using the interactions GXPF1B and SDPFMU-DB [30]. For other calculations of ${ }^{48} \mathrm{Ca}$ using the shell model or the methods related to this model, see Refs. $[31,39,40,43,49-53]$ and the references cited therein. See also Refs. [34,54] for other QRPA calculations for the nucleus.

Here, we describe the technical aspects of our QRPA calculations. The particle-hole interaction is the Skyrme (parameter set $\mathrm{SkM}^{*}$ [55]), and the pairing interaction is the contact interaction with no density dependence. The strength of the pairing interaction was determined to reproduce the pairing gap deduced from the mass data using the three-point formula [56] with a very low cutoff occupation probability for the canonical single-particle states in the paired case or a very high cutoff energy in the unpaired case in the Hartree-Fock-Bogoliubov calculations. These are the pairing interactions for like-particles. The isoscalar and isovector proton-neutron pairing interactions were also used for the QRPA calculation; for these inter- 


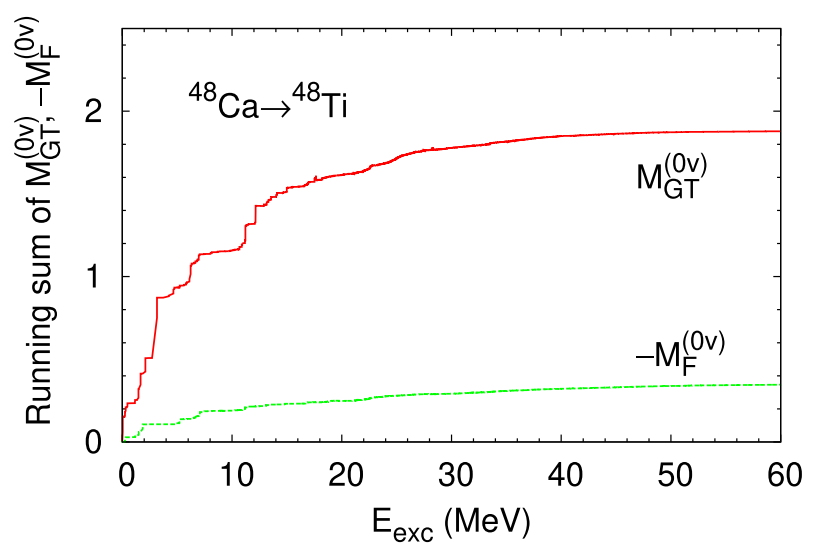

Fig. 1 Running sums of $M_{\mathrm{GT}}^{(0 v)}$ and $M_{\mathrm{F}}^{(0 v)}$ for ${ }^{48} \mathrm{Ca} \rightarrow{ }^{48} \mathrm{Ti}$ obtained by the QRPA [48] as functions of the excitation energy $E_{\text {exc }}$ of the intermediate nucleus ${ }^{48} \mathrm{Sc}$. The actual calculations were performed up to approximately $75 \mathrm{MeV}$. No quenching factor was used

actions, see Ref. [57] and the references therein. The calculations were performed using the $\mathrm{M}$ scheme. The number of single-particle levels used for the QRPA calculation was approximately 1700 for each of the protons and neutrons, that is, the valence single-particle space. The total number of QRPA solutions was nearly 600000 for both ${ }^{48} \mathrm{Ca}$ and ${ }^{48} \mathrm{Ti}$. For details of the calculation, see Ref. [48].

We obtained the running sums (cumulation) of $M_{\mathrm{GT}}^{(0 v)}$ and $M_{\mathrm{F}}^{(0 v)}$ from the QRPA solutions for ${ }^{48} \mathrm{Ca} \rightarrow{ }^{48} \mathrm{Ti}$ [48], as shown in Fig. 1. The horizontal axis indicates the excitation energy $E_{\text {exc }}$ of the intermediate nucleus ${ }^{48} \mathrm{Sc}$. The two NME components converge around $E_{\text {exc }}$ $=50 \mathrm{MeV}$. The first step of our approach is to compare the energy dependence of this running sum with the results of the shell model calculation using the truncated valence singleparticle spaces. The shell model is usually applied to the $0 \nu \beta \beta$-NME calculation without the intermediate states. It is necessary to consider the effective maximum $E_{\text {exc }}$ of the shell model calculations. Let us consider a simple example that only two single-particle levels are involved in the shell model. If the one-particle one-hole (1p1h) energy is $5 \mathrm{MeV}$, and the two singleparticle levels have a ten-fold degeneracy, the $10 \mathrm{p} 10 \mathrm{~h}$ energy is $50 \mathrm{MeV}$ under the assumption that the low level is fully occupied in the lowest-energy configuration. Comparison of the NME of this shell model and the QRPA running NME at $E_{\text {exc }}=50 \mathrm{MeV}$ would not be useful because the low-order particle-hole excitations of $50 \mathrm{MeV}$ are not included in the shell model calculation. Thus, the energy of the shell model corresponding to the $E_{\text {exc }}$ of the QRPA is not a trivial question. One method to address this question is to refer to the charge-change transition strength with the angular momentum and the parity of $J^{\pi}=1^{+}$of the shell model and the experimental data.

For the shell model, as shown in Fig. 2, the authors of Ref. [58] fitted the experimental charge-change strength function of ${ }^{48} \mathrm{Ca}$ and ${ }^{48} \mathrm{Ti}$ by a quenching factor of 0.77 to the GT operator in the $p f$ valence shell calculation; the quenching factor to the strength function is 0.59 . In our observation, their fitting is good up to $13 \mathrm{MeV}$ for ${ }^{48} \mathrm{Ca}$ and $7.5 \mathrm{MeV}$ for ${ }^{48} \mathrm{Ti}$. The NME of the double- $\beta$ decay requires the products of the transition densities of ${ }^{48} \mathrm{Ca} \rightarrow{ }^{48} \mathrm{Sc}$ and ${ }^{48} \mathrm{Ti} \rightarrow{ }^{48} \mathrm{Sc}$. Thus, it is inferred that the shell model with the $p f$ valence shell is reliable for the components of $M_{\mathrm{GT}}^{(0 v)}$ of ${ }^{48} \mathrm{Ca}$ up to $E_{\mathrm{exc}}=7.5 \mathrm{MeV}$. Because the valence single-particle space is essential for determination of the reliable energy region, we 

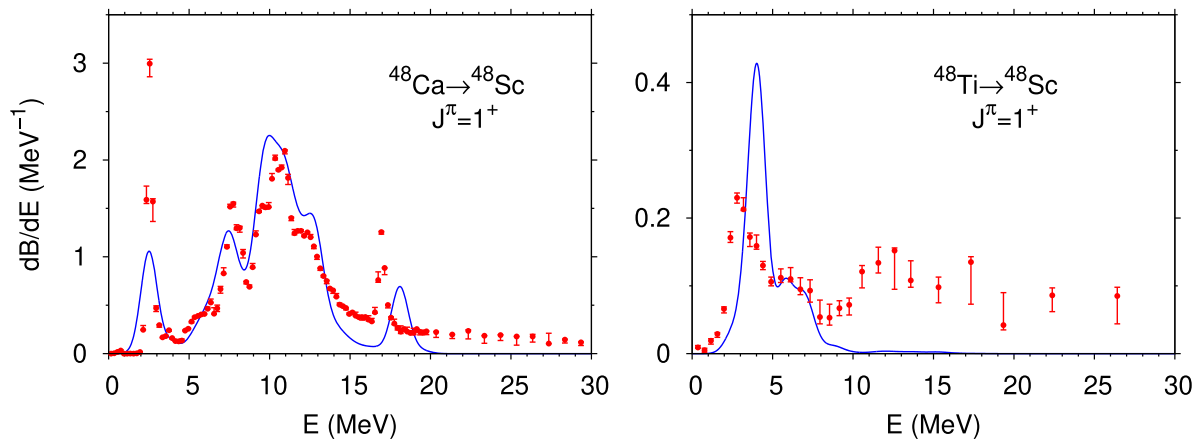

Fig. 2 Charge-change strength function $d B / d E$ with $J^{\pi}=1^{+}$based on a shell model calculation (blue curves) accounting for the $p f$ valence shell (interaction GXPF1B) and experimental data (red bars) [59] as functions of excitation energy of ${ }^{48} \mathrm{Sc}$. The left (right) figure shows the strength function from ${ }^{48} \mathrm{Ca}\left({ }^{48} \mathrm{Ti}\right)$ to ${ }^{48} \mathrm{Sc}$. For the calculation, the $\mathrm{GT}^{-}\left(\mathrm{GT}^{+}\right)$operator $\sigma \tau^{-}\left(\sigma \tau^{-\dagger}\right)$ with a quenching factor 0.77 was used for the left (right) figure. For the experimental data, the possibility is pointed out [59] that the isovector spin monopole operator is involved in addition to the GT operator. The Gaussian function is applied to the calculation with its square root of the variance: $\sigma=0.510 \mathrm{MeV}$. The experimental data have full widths at half maximum of energy resolution of $0.2 \mathrm{MeV}$, corresponding to $\sigma=0.085 \mathrm{MeV}$, in the left figure, and $0.4 \mathrm{MeV}$, corresponding to $\sigma=0.170 \mathrm{MeV}$, in the right figure

assume that this region does not change appreciably for other not-very-high angular momenta and parity, as long as the same valence single-particle space is used.

A question arises as to why the quenching factor is necessary if the shell model is reliable. One of the reasons is the necessity to add the isovector spin monopole (IVSM) operator, i.e., the GT operator multiplied by $r_{1}^{2}$ ( $r_{1}$ is the radial variable of a nucleon), to the transition operator, as pointed out in Ref. [59]. The discussion in this section is not affected by the IVSM operator. However, the following point is worth noting: if the IVSM operator is used, the missing transition strength in $E_{\text {exc }}>7.5 \mathrm{MeV}$ in ${ }^{48} \mathrm{Ti} \rightarrow{ }^{48} \mathrm{Sc}$ would not be reproduced in the present shell model calculations with the $s d p f$ valence shell. The two-major-shell jump is necessary for activating the high-energy components of the IVSM operator $[48,60]$; thus, the $s d p f$ valence shell is not sufficient. An analogous discussion is applied to the tail region of the strength function of ${ }^{48} \mathrm{Ca} \rightarrow{ }^{48} \mathrm{Sc}$. It is possible that the transition operator should be also modified by many-body effects, as mentioned in the introduction in relation to the effective $g_{A}$.

The $M_{\mathrm{GT}}^{(0 v)}$ of the QRPA is 1.14 at $E_{\mathrm{exc}}=7.5 \mathrm{MeV}$, and the converged value is 1.88 ; these values are summarized in Table 1 . The shell model should include the $1 \mathrm{p} 1 \mathrm{~h}$ correlations of the QRPA in any energy region, if the valence single-particle space is sufficiently large. Thus, the increasing behavior of $M_{\mathrm{GT}}^{(0 v)}$ and $M_{\mathrm{F}}^{(0 v)}$ of the QRPA should be included in the very large shell model calculations. We estimate the effect of the missing valence single-particle space in the current shell model based on the ratio of the converged $M_{\mathrm{GT}}^{(0 v)}$ of the QRPA to the running sum up to $E_{\mathrm{exc}}=7.5 \mathrm{MeV}$. The components of the $0 \nu \beta \beta$ NME of the shell model are listed in Table 2. We use the average values of the two short-range correlations (SRC): CD-Bonn and Argonne. The average $M_{\mathrm{GT}}^{(0 v)}$ of the $p f$ valence-shell calculation is 0.77 , and that for the $s d p f$ valence shell is 1.00 . The converged $M_{\mathrm{GT}}^{(0 v)}$ of the shell model is estimated to be the shell model value multiplied by the increasing ratio of the QRPA, that is, 
Table 1 Partial $M_{\mathrm{GT}}^{(0 v)}$ of the QRPA at $E_{\text {exc }}=7.5 \mathrm{MeV}$ in the running sum and the converged value for ${ }^{48} \mathrm{Ca}$

\begin{tabular}{ll}
\hline$E_{\text {exc }}(\mathrm{MeV})$ & $M_{\mathrm{GT}}^{(0 v)}(\mathrm{QRPA})$ \\
\hline 7.5 & 1.14 \\
$\sim 70$ & 1.88
\end{tabular}

Table 2 Components of $M_{\mathrm{GT}}^{(0 v)}$ and $M_{\mathrm{F}}^{(0 v)}$ of the shell model for ${ }^{48} \mathrm{Ca}$ [30]. We show the results with the interactions GXPF1B for the $p f$ valence shell and SDPFMU-DB for the $s d p f$ valence shell. Only the results necessary for our discussion are referred to. SRC denotes the short-range correlation, and CD-Bonn and Argonne are used. No quenching factor was used

\begin{tabular}{|c|c|c|c|c|}
\hline \multirow[t]{2}{*}{ SRC } & \multicolumn{2}{|l|}{$p f$} & \multicolumn{2}{|l|}{$\underline{s d p f}$} \\
\hline & $M_{\mathrm{GT}}^{(0 v)}$ & $M_{\mathrm{F}}^{(0 v)}$ & $M_{\mathrm{GT}}^{(0 v)}$ & $M_{\mathrm{F}}^{(0 v)}$ \\
\hline None & 0.776 & -0.216 & 0.997 & -0.304 \\
\hline CD-Bonn & 0.809 & -0.233 & 1.045 & -0.327 \\
\hline Argonne & 0.743 & -0.213 & 0.953 & -0.300 \\
\hline
\end{tabular}

The underlying assumption for this estimation is that the effects of the mpmh correlations increase with the same ratio as the $1 \mathrm{p} 1 \mathrm{~h}$ effects. This assumption is justified by the method discussed in the next section.

\subsection{Method with the help of single-particle energy}

Another simple way to compare the shell model and QRPA calculations is to identify the largest 1 p 1 h energy of the truncated valence single-particle space with $E_{\text {exc }}$ in Fig. 1 . The single-particle energies of ${ }^{48} \mathrm{Ca}$ are shown in Table 3 obtained using an updated Woods-Saxon potential [61]. We use this potential because it is a very realistic single-particle potential. The largest $1 \mathrm{p} 1 \mathrm{~h}$ energy in the neutron $p f$ valence shell is $8.8 \mathrm{MeV}\left(1 f_{5 / 2}-1 f_{7 / 2}\right)$, and that of the $s d p f$ valence shell is $14.4 \mathrm{MeV}\left(1 f_{5 / 2}-1 d_{5 / 2}\right)$. The corresponding largest $1 \mathrm{p} 1 \mathrm{~h}$ energies of the proton are $4.71 \mathrm{MeV}\left(2 p_{1 / 2}-1 f_{7 / 2}\right)$ and $16.83 \mathrm{MeV}\left(2 p_{1 / 2}-1 d_{5 / 2}\right)$, respectively. The largest $1 \mathrm{p} 1 \mathrm{~h}$ energy in the neutron $p f$ valence shell is similar to the $E_{\mathrm{exc}}=7.5 \mathrm{MeV}$ of the $p f$ valence-shell calculation discussed above. Thus, the two methods used to derive the effective $E_{\text {exc }}$ of the shell model are approximately consistent. We assume that the single-particle energies are not appreciably different for ${ }^{48} \mathrm{Ca}$ and ${ }^{48} \mathrm{Sc}$.

The $M_{\mathrm{GT}}^{(0 v)}$ values of the shell model are summarized in Table 4, and $M_{\mathrm{GT}}^{(0 v)}$ values of the QRPA at $E_{\text {exc }}$ corresponding to the largest $1 \mathrm{p} 1 \mathrm{~h}$ energies of the truncated valence singleparticle space are summarized in Table 5. In the application of the $p f$ valence shell model to ${ }^{48} \mathrm{Ca}$, the role of the protons is either small or none. Thus, we refer to the neutron excitation energy to compare the shell model and QRPA calculations. The ratio of two $M_{\mathrm{GT}}^{(0 v)}$ ( $s d p f$ to $p f$ ) of the QRPA, 1.31, shown in Table 5 is consistent with the corresponding ratio of the shell model (1.30), as shown in Table 4. This implies that the relative energy dependence of the mpmh effects beyond $1 \mathrm{p} 1 \mathrm{~h}$ is close to that of the $1 \mathrm{p} 1 \mathrm{~h}$ effects. In addition, the components of the NME of the QRPA order should be included in the very large valence shell model, as mentioned before. These two points justify our approach.

Now, we can estimate the converged value of $M_{\mathrm{GT}}^{(0 v)}$ of the shell model two ways (see Tables 4 and 5) as 
Table 3 Neutron (left) and proton (right) single-particle energies associated with ${ }^{48} \mathrm{Ca}$ obtained from the Woods-Saxon potential [61]

\begin{tabular}{llll}
\hline Orbital & Single-particle energy $(\mathrm{MeV})$ & Orbital & Single-particle energy $(\mathrm{MeV})$ \\
\hline Neutron hole & & Proton hole & \\
$1 d_{5 / 2}$ & -15.61 & $1 d_{5 / 2}$ & -21.47 \\
$2 s_{1 / 2}$ & -12.55 & $1 d_{3 / 2}$ & -16.18 \\
$1 d_{3 / 2}$ & -12.53 & $2 s_{1 / 2}$ & -16.10 \\
$1 f_{7 / 2}$ & -10.00 & & \\
Neutron particle & & Proton particle & \\
$2 p_{3 / 2}$ & -4.60 & $1 f_{7 / 2}$ & -9.35 \\
$2 p_{1 / 2}$ & -2.86 & $2 p_{3 / 2}$ & -6.44 \\
$1 f_{5 / 2}$ & -1.20 & $2 p_{1 / 2}$ & -4.64 \\
$1 g_{9 / 2}$ & 0.130 & &
\end{tabular}

Table $4 M_{\mathrm{GT}}^{(0 v)}$ of the shell model with two different valence single-particle spaces and the ratio of $M_{\mathrm{GT}}^{(0 v)}$ to that of the $p f$ valence-shell calculation

\begin{tabular}{lll}
\hline Shell & $M_{\mathrm{GT}}^{(0 v)}$, shell model & Ratio to $M_{\mathrm{GT}}^{(0 v)}(p f)$ \\
\hline$p f$ & 0.77 & 1.0 \\
sdpf & 1.00 & 1.30 \\
\hline
\end{tabular}

Table $5 M_{\mathrm{GT}}^{(0 v)}$ of the QRPA at $E_{\text {exc }}$ corresponding to the largest $1 \mathrm{p} 1 \mathrm{~h}$ energy of the truncated valence singleparticle space (maximum $E_{\mathrm{exc}}$ ). The ratio of $M_{\mathrm{GT}}^{(0 v)}$ to that of the $p f$ valence-shell equivalent calculation is also shown. The last row shows the converged result

\begin{tabular}{llll}
\hline Equivalent shell & Max $E_{\mathrm{exc}}(\mathrm{MeV})$, neutrons & $M_{\mathrm{GT}}^{(0 v)}, \mathrm{QRPA}$ & Ratio of $M_{\mathrm{GT}}^{(0 v)}$ to $M_{\mathrm{GT}}^{(0 v)}(p f)$ \\
\hline$p f$ & 8.8 & 1.15 & 1.0 \\
$s d p f$ & 14.4 & 1.51 & 1.31 \\
& $\sim 70$ & 1.88 & 1.63 \\
\hline
\end{tabular}

Table 6 Original $M_{\mathrm{GT}}^{(0 v)}$ of the shell model and estimated values corresponding to a very large valence single-particle space

\begin{tabular}{lll}
\hline Original shell & Original $M_{\mathrm{GT}}^{(0 v)}$, shell model & Estimate of converged $M_{\mathrm{GT}}^{(0 v)}$ \\
\hline$p f$ & 0.77 & 1.26 \\
sdpf & 1.00 & 1.25 \\
\hline
\end{tabular}

$$
1.00 \cdot(1.88 / 1.51)=1.25 \text {, extension from the } s d p f \text { shell. }
$$

The two estimated values and the value of Eq. (6) are distributed within a narrow region. The results of the second method are summarized in Table 6 . The estimated converged value is $64 \%$ larger than the $p f$ valence-shell value and $25 \%$ larger than the $s d p f$ valence-shell value. 


\section{Modification of GT component of $0 v \beta \beta$ NME of QRPA}

Next, we estimate the mpmh effects and modify the QRPA NME. In this section, $M_{\mathrm{GT}}^{(0 v)}$ indicates that of the QRPA. In principle, the best method is to compare experimental data involving the charge-change transition density and the result of the QRPA, and one possible candidate is the data of the charge-change strength functions of the $1^{+}$states in Fig. 2. However, this scheme is not straightforward because the transition operator causing the data of the strength functions is not clearly known. This complexity is not surprising because the charge exchange reaction occurs as a result of the nuclear force. As mentioned previously, this transition operator has an IVSM component. The mixing ratio of the GT and IVSM operators is not known a priori.

In this study, we use a simple method to evaluate the mpmh effect. This refers to the quenching factor previously known for the strength function of the shell model with the GT operator to reproduce the data. In Ref. [58], a quenching factor of 0.77 is applied to the GT operator for both $p f$ and $s d p f$ valence-shell calculations, as mentioned before. Conversely, the quenching factors of the QRPA calculation for the GT strength function are 0.5 for ${ }^{48} \mathrm{Ca} \rightarrow{ }^{48} \mathrm{Sc}$ and 0.38 for ${ }^{48} \mathrm{Ti} \rightarrow{ }^{48} \mathrm{Sc}$ [48]. These factors were then determined in low-energy regions similar to those discussed for the shell model. There are two origins of the quenching factor in the QRPA transition strength. One is the effect of the mpmh correlations missing in the QRPA nuclear wave functions, and the other is the modification of the transition operator, e.g., the IVSM components and vertex corrections. It is known that the mpmh correlations reduce the charge-change transition strength in low-energy regions [62-64] and the NME [65]. The shell model requires only the quenching factor caused by the operator modification. The quenching factor for the QRPA is the product of the factors from the two origins, and the one caused by the operator modification is shared by the two methods. For ${ }^{48} \mathrm{Ca}$, this idea leads to a relation between the quenching factors in the strength functions

$$
0.5=0.77^{2} x,
$$

where $x$ is the quenching factor related to the mpmh correlations of the nuclear wave functions; for ${ }^{48} \mathrm{Ti}$, we have

$$
0.38=0.77^{2} x^{\prime}
$$

where $x^{\prime}$ is the same as $x$ but for ${ }^{48} \mathrm{Ti}$. Thus, we obtain $x=0.847$ and $x^{\prime}=0.644$. The difference between these two values indicates that the mpmh effect is larger in ${ }^{48} \mathrm{Ti}$ than it is in ${ }^{48} \mathrm{Ca}$. The QRPA is a reasonable approximation to the doubly magic ${ }^{48} \mathrm{Ca}$; however, the quality of approximation deteriorates for ${ }^{48} \mathrm{Ti}$. Thus, the $0 \nu \beta \beta$ NME is affected. The quenching factors are applied in the low-energy regions where the shell model is reliable.

We introduce four modification factors to $\left\langle F\left|c_{p}^{\dagger} c_{n}\right| B_{F}\right\rangle$ and $\left\langle B_{I}\left|c_{p^{\prime}}^{\dagger} c_{n^{\prime}}\right| I\right\rangle$ when they are used with the GT operator [see Eq. (5)]:

- the quenching factor $q_{l}^{F}$ multiplied by $\left\langle F\left|c_{p}^{\dagger} c_{n}\right| B_{F}\right\rangle$ with $\left|B_{F}\right\rangle$ in the low-energy region corresponding to the reliable region of the shell model with the one major valence shell,

- the enhancing factor $q_{h}^{F}$ multiplied by the same transition density matrix element but for the high-energy region above the low-energy region, and

- $q_{l}^{I}$ and $q_{h}^{I}$ which are the same as $q_{l}^{F}$ and $q_{h}^{F}$ but for $\left\langle B_{I}\left|c_{p^{\prime}}^{\dagger} c_{n^{\prime}}\right| I\right\rangle$, respectively.

Enhancing factors are introduced for the high-energy region because of the sum rule of the transition strength. The modification of the transition density matrix is shared by the strength function associated with the charge exchange reaction and the NME of the double- $\beta$ decay involving the spin transition operator. 
The partitioning of the energy region and the quenching factor in the low-energy region were determined based on the smoothed strength functions. Therefore, the effects of mpmh on the $0 \nu \beta \beta$ NME should be simulated in the same manner. With these modification factors, smoothening the intermediate-state energy dependence by the Lorentzian function, and truncation up to the first order with respect to $(1-q)$ ( $q$ is any of the four modification factors), the following equation for the modified $M_{\mathrm{GT}}^{(0 v)}$ is obtained:

$$
\begin{aligned}
M_{\mathrm{GT}}^{(0 v)}(\text { modified })= & M_{\mathrm{GT}}^{(0 v)}+M_{\mathrm{GT}-1 l}^{(0 v)}+M_{\mathrm{GT}-1 h}^{(0 v)}, \\
M_{\mathrm{GT}-1 l}^{(0 v)}= & -\left(1-q_{l}^{F}\right) \frac{1}{\pi} \int_{-\infty}^{E_{c}^{F}} d E \sum_{E_{B F}} \frac{\varepsilon P_{F}\left(E_{B F}\right)}{\left(E_{B F}-E\right)^{2}+\varepsilon^{2}} \\
& -\left(1-q_{l}^{I}\right) \frac{1}{\pi} \int_{-\infty}^{E_{c}^{I}} d E \sum_{E_{B I}} \frac{\varepsilon P_{I}\left(E_{B I}\right)}{\left(E_{B I}-E\right)^{2}+\varepsilon^{2}}, \\
M_{\mathrm{GT}-1 h}^{(0 v)}= & -\left(1-q_{h}^{F}\right) \frac{1}{\pi} \int_{E_{c}^{F}}^{\infty} d E \sum_{E_{B F}} \frac{\varepsilon P_{F}\left(E_{B F}\right)}{\left(E_{B F}-E\right)^{2}+\varepsilon^{2}} \\
& -\left(1-q_{h}^{I}\right) \frac{1}{\pi} \int_{E_{c}^{I}}^{\infty} d E \sum_{E_{B I}} \frac{\varepsilon P_{I}\left(E_{B I}\right)}{\left(E_{B I}-E\right)^{2}+\varepsilon^{2}}, \\
P_{F}\left(E_{B F}\right)= & \sum_{B_{I}} \sum_{p n p^{\prime} n^{\prime}}\left\langle p p^{\prime}\left|V_{\mathrm{GT}}^{(0 v)}\left(r ; \bar{E}_{B}\right)\right| n n^{\prime}\right\rangle\left\langle F\left|c_{p}^{\dagger} c_{n}\right| B_{F}\right\rangle\left\langle B_{F} \mid B_{I}\right\rangle\left\langle B_{I}\left|c_{p^{\prime}}^{\dagger} c_{n^{\prime}}\right| I\right\rangle, \\
P_{I}\left(E_{B I}\right)= & \sum_{B_{F}} \sum_{p n p^{\prime} n^{\prime}}\left\langle p p^{\prime}\left|V_{\mathrm{GT}}^{(0 v)}\left(r ; \bar{E}_{B}\right)\right| n n^{\prime}\right\rangle\left\langle F\left|c_{p}^{\dagger} c_{n}\right| B_{F}\right\rangle\left\langle B_{F} \mid B_{I}\right\rangle\left\langle B_{I}\left|c_{p^{\prime}}^{\dagger} c_{n^{\prime}}\right| I\right\rangle,(15)
\end{aligned}
$$

where $E_{B I}$ and $E_{B F}$ are the QRPA eigenenergies of the $\left|B_{I}\right\rangle$ and $\left|B_{F}\right\rangle$, respectively. The parameter $\varepsilon>0$ is a constant chosen to reproduce the width of the experimental strength function. $E_{c}^{I}$ and $E_{c}^{F}$ are the QRPA energies distinguishing the low- and high-energy regions associated with the initial and final states, respectively. The lowest QRPA energy of the transition to the intermediate nucleus is identified with the transition energy to the ground state of the intermediate nucleus. $E_{c}^{I}$ and $E_{c}^{F}$ are obtained by adding these lowest QRPA energies to the boundary excitation energies discussed above. $E_{c}^{I}=12.053 \mathrm{MeV}$ and $E_{c}^{F}=$ 17.387 MeV were obtained from our numerical calculations. The deviation of the modification factors from unity represents the modification effects. Thus, if there is no modification, the modification terms vanish. Because the quenching factors are less than one, these factors decrease the NME in the low-energy region. The enhancing factors increase the NME in the high-energy region.

We use

$$
\begin{aligned}
q_{l}^{I} & =\sqrt{x}=0.92, \text { and } \\
q_{l}^{F} & =\sqrt{x^{\prime}}=0.80 .
\end{aligned}
$$

The transition strength up to $E_{\text {exc }}=13 \mathrm{MeV}$ is 19.575 for ${ }^{48} \mathrm{Ca} \rightarrow{ }^{48} \mathrm{Sc}$ (the integral of the QRPA strength function). The redundant transition strength in $E_{\mathrm{exc}}<13 \mathrm{MeV}$ due to the insufficiency of the mpmh correlations is estimated as

$$
19.575(1-x)=2.99 \text {. }
$$


The transition strength in the high-energy region is 4.96 . The enhancing factor is calculated as

$$
q_{h}^{I}=\sqrt{(2.99+4.96) / 4.96}=1.27 .
$$

In the same manner, we obtain $q_{h}^{F}=1.44$ for ${ }^{48} \mathrm{Ti}$.

We discuss the uncertainty of our approach and further modifications of the equation for the QRPA NME. The mpmh correlation has two effects. One is to modify the QRPA states, and the other is to create mpmh states that are not included in the QRPA. When the GT strength in the low-energy region is reduced by the mpmh correlations, the absolute values of the major transition density matrix elements of the QRPA states must decrease sufficiently, overwhelming the effect of the mpmh states of increasing the strength. Thus, the reduction in the contribution of the QRPA states to the NME is the main effect of the modification in the low-energy region. The sum rule implies that a shift in the strength of the transition density matrix elements occurs from the low- to the high-energy region. This shift enhances the contribution of the QRPA states to the $0 \nu \beta \beta$-decay NME in that region, if the change occurs uniformly with respect to the matrix elements. Equation (11) expresses this effect.

The effect of the mpmh states is not negligible in the high-energy region. In the continuum region, it is known as the spreading width, e.g., [66]. In reality, the importance of the mpmh-state effects increases gradually as the excitation energy increases. We introduce a simplification wherein this effect is significant only in the high-energy region. The contribution of the mpmh states to the NME of the double- $\beta$ decay cannot be estimated by our approach because this specific effect may reduce or enhance the NME. The sign of the contribution is unknown without the calculations including the mpmh effects in the high-energy regions.

Equation (11) expresses an extreme case in which the mpmh corrections in the high-energy region arise entirely through the modification of the QRPA states; we call this case extreme case I. It is possible to consider another extreme case in which the mpmh corrections in the high-energy region are entirely carried by the new states beyond the QRPA; we call this case extreme case II. Three sub-extreme cases belonging to extreme case II can be discussed as follows.

i. The mpmh-state corrections are maximally coherent with $M_{\mathrm{GT}}^{(0 v)}$. Usually, the effects of the mpmh states are smaller than those of the $1 \mathrm{p} 1 \mathrm{~h}$ states, e.g., for the transition strength. Thus, $M_{\mathrm{GT}}^{(0 v)}$ in this extreme case may not exceed $M_{\mathrm{GT}}^{(0 v)}$ (modified) of Eq. (11) derived in extreme case I. Thus, Eq. (11) gives the upper limit for extreme case II.

ii. The mpmh-state corrections are maximally anticoherent to $M_{\mathrm{GT}}^{(0 v)}$. In this case, the lower limit of $M_{\mathrm{GT}}^{(0 v)}$ (modified) is estimated

$$
M_{\mathrm{GT}}^{(0 v)}+M_{\mathrm{GT}-1 l}^{(0 v)}-M_{\mathrm{GT}-1 h}^{(0 v)} .
$$

iii. The mpmh-state corrections are accompanied by strong randomness, and the corrections are canceled. In this extreme case, $M_{\mathrm{GT}}^{(0 v)}$ (modified) is given by

$$
M_{\mathrm{GT}}^{(0 v)}+M_{\mathrm{GT}-1 l}^{(0 v)} .
$$

Thus, we can evaluate the uncertainty range of $M_{\mathrm{GT}}^{(0 v)}$ (modified) as

$$
M_{\mathrm{GT}}^{(0 v)}+M_{\mathrm{GT}-1 l}^{(0 v)}-M_{\mathrm{GT}-1 h}^{(0 v)}<M_{\mathrm{GT}}^{(0 v)}(\text { modified })<M_{\mathrm{GT}}^{(0 v)}+M_{\mathrm{GT}-1 l}^{(0 v)}+M_{\mathrm{GT}-1 h}^{(0 v)} .
$$

The $M_{\mathrm{GT}}^{(0 v)}$ (modified) of extreme case I is equal to the upper limit of extreme case II. It is speculated that the upper limit in the mixing case between the two extreme cases does not 


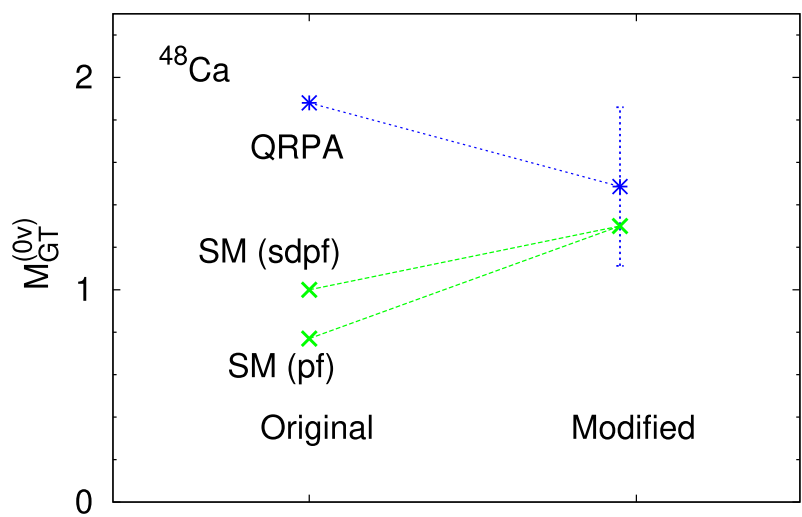

Fig. 3 Values of $M_{\mathrm{GT}}^{(0 v)}$ of the shell model and QRPA, labeled "Original", and the modified $M_{\mathrm{GT}}^{(0 v)}$, labeled "Modified". The shell model, denoted by SM, and QRPA are distinguished by different symbols. The modified shell model value is the average of $1.25,1.26$, and 1.27 obtained in Sect. 3. The error bar of the modified QRPA shows the uncertainty range, and the mean value is interpreted as the value in the randomness limit (see text)

significantly exceed the upper limit of Eq. (22). If one effect is weakened, and another effect appears, the total may not change significantly. The total effect in the high-energy region is restricted because the change in the transition density is restricted by the sum rule. A similar discussion is possible for the lower limit in the mixing case. $M_{\mathrm{GT}-1 l}^{(0 v)}=-0.394$ and $M_{\mathrm{GT}-1 h}^{(0 v)}$ $=0.374$ are obtained numerically; consequently, the uncertainty range is obtained as

$$
1.112<M_{\mathrm{GT}}^{(0 v)}(\text { modified })<1.860,
$$

by referring to the value of $M_{\mathrm{GT}}^{(0 v)}$ of the QRPA in Sect. 3. $M_{\mathrm{GT}}^{(0 v)}$ (modified) in the limit of randomness of case iii is 1.486. The results are summarized in Fig. 3. The modified QRPA value in the randomness limit and the converged value of the shell model based on our estimation are much close compared with the original values of the two models. The mpmh correlations are generally accompanied by randomness in the high-energy regions. This tendency is indicated by, e.g., the success of the random matrix theory $[56,67]$. Therefore, we speculate that the best modified value of the QRPA is closer to the randomness limit than the edge values of the coherent limit. Our results show that the consistency of the shell model and the QRPA can be obtained by taking into account the sufficiently large valence single-particle space and the mpmh correlations.

\section{Fermi component of $0 v \beta \beta$ NME}

According to Ref. [30], the $M_{\mathrm{F}}^{(0 v)}$ of the $p f$ valence-shell calculation averaged for the two SRC methods is -0.223 , and that of the $s d p f$ valence-shell calculation is -0.314 , where no quenching factor is used. The latter is $41 \%$ larger than the former in terms of the absolute value. The corresponding increasing ratio of the QRPA read from Fig. 1 is $21 \%$; the $M_{\mathrm{F}}^{(0 v)}$ value is -0.19 at $E_{\text {exc }}=8.8 \mathrm{MeV}$, corresponding to the $p f$ valence shell, and -0.23 at $E_{\text {exc }}=$ 14.4 MeV, corresponding to the $s d p f$ valence shell. These equivalent $E_{\text {exc }}$ values are listed in Table 5. There is a non-negligible difference in the increasing ratios of the two methods. The converged value of $M_{\mathrm{F}}^{(0 v)}$ of the QRPA is -0.35 , of which the absolute value is increased from 
Table $7 \quad M_{\mathrm{F}}^{(0 v)}$ of the shell model for two different valence shells and those of the QRPA for the equivalent $E_{\text {exc }}$ 's and a very large $E_{\text {exc }}$

\begin{tabular}{lccc}
\hline Shell & Equivalent $\max E_{\mathrm{exc}}(\mathrm{MeV})$ & $M_{\mathrm{F}}^{(0 v)}($ shell model $)$ & $M_{\mathrm{F}}^{(0 v)}(\mathrm{QRPA})$ \\
\hline$p f$ & 8.8 & -0.223 & -0.19 \\
sdpf & 14.4 & -0.314 & -0.23 \\
Large-space limit & & & -0.35 \\
\hline
\end{tabular}

the value at $14.4 \mathrm{MeV}$ by $52 \%$. The $M_{\mathrm{F}}^{(0 v)}$ values are listed in Table 7 . The absolute value of $M_{\mathrm{F}}^{(0 v)}$ of the shell model is larger than that of the QRPA with the same $E_{\text {exc }}$. This relation of magnitude is inverted in comparison with that for $M_{\mathrm{GT}}^{(0 v)}$ (see Tables 5 and 6). According to the method applied to $M_{\mathrm{GT}}^{(0 v)}$ in Sect. 3, we obtain two estimates for the extrapolated $M_{\mathrm{F}}^{(0 v)}$ of the shell model:

$$
\begin{aligned}
& -0.223 \cdot(0.35 / 0.19)=-0.411, \text { and } \\
& -0.314 \cdot(0.35 / 0.23)=-0.478,
\end{aligned}
$$

(see Table 7).

To consider $M_{\mathrm{F}}^{(0 v)}$ of the QRPA, we discuss the transition operator dependence of the modification factor from a general viewpoint. To our knowledge, this dependence can be summarized as follows:

- Appreciable quenching is necessary for the GT transition, whether it is caused by the strong or weak interaction, at least for the transitions with non-high transition energies corresponding to a one major valence shell (see Sect. 4) or spin-orbit splitting. Both the QRPA and shell model require the quenching factor; the appropriate value depends on method.

- The isobaric-analog transition does not require a quenching factor at least for the QRPA. This is not surprising because the Fermi transition strength concentrates on the isobaricanalog state, e.g., [68], and the QRPA satisfies the Fermi sum rule.

- For the electric transitions, the necessity of the effective charge is much lower than that of the quenching factor for the GT operator except for maintaining the center of mass, e.g., [69]. This is particularly clear for the nuclei to which the QRPA is a good approximation.

- Magnetic transition requires an effective $g$ factor, e.g., [37,70]. Significant adjustment of the spin $g$ factor is necessary.

When these features are stated for the QRPA, sufficiently large single-particle spaces are assumed. From this observation, we obtain the idea that the transition operator is essential for the modification factors rather than the difference in the interactions, that is,

- the spin operator has a high necessity for the quenching factor at least for transitions with non-high energies.

- Coordinate operators do not have that necessity at least for the nuclei to which the QRPA is a good approximation.

- The operator only changing the charge does not require quenching.

Based on the last two items, we do not quench the $M_{\mathrm{F}}^{(0 v)}$ of the QRPA. 
Table 8 Estimated $M_{\mathrm{GT}}^{(0 v)}$ and $M_{\mathrm{F}}^{(0 v)}$ from the shell model and the QRPA

\begin{tabular}{lll}
\hline Component & Estimate from shell model & Estimate from QRPA \\
\hline$M_{\mathrm{GT}}^{(0 v)}$ & 1.26 & 1.49 \\
$-M_{\mathrm{F}}^{(0 v)}$ & $0.41-0.48$ & 0.35 \\
\hline
\end{tabular}

\section{Estimated $0 v \beta \beta$ NME}

$M^{(0 v)}$ can be calculated using the modified components. We did not pinpoint the modified components of $M^{(0 v)}$ of the QRPA because of the uncertainty of our estimation. In this section, we make a choice to simplify the comparison. We use the QRPA $M_{\mathrm{GT}}^{(0 v)}$ (modified) obtained with the complete randomness of the mpmh correlations in the high-energy region (see Sect. 4). For the Fermi component of the shell model, we do not have a speculation on what value is more likely than others in the range of $-M_{\mathrm{F}}^{(0 v)}=0.411-0.478$. The modified NME components of the shell model and the QRPA are summarized in Table 8.

By using $g_{V}=1.0$, and tentatively the bare value of $g_{A}=1.276$ [36], the estimated $M^{(0 v)}$ based on the shell model for ${ }^{48} \mathrm{Ca}$ is found to be $1.512-1.554$, and that from the QRPA is 1.705. A partial cancelation occurs between the difference in $M_{\mathrm{GT}}^{(0 v)}$ of the two methods and that in $M_{\mathrm{F}}^{(0 v)}$ of these methods, as shown in Table 8. The values of 1.5-1.7 are situated in the middle of the distribution of $M^{(0 v)}$ using various methods; see Ref. [28], in which the $M^{(0 v)}$ values are in the range of 0.6 to 3.0 for ${ }^{48} \mathrm{Ca}$.

\section{NME of $2 v \beta \beta$ decay}

Next, the physical quantities to be examined are the components of the $2 \nu \beta \beta$ NME $M_{\mathrm{GT}}^{(2 v)}$ and $M_{\mathrm{F}}^{(2 v)}$; the former is the GT component, and the latter is the Fermi component. The former is defined by

$$
M_{\mathrm{GT}}^{(2 v)}=\sum_{B} \frac{1}{E_{B}-\bar{M}}\left\langle F\left|\sigma \tau^{-}\right| B\right\rangle \cdot\left\langle B\left|\sigma \tau^{-}\right| I\right\rangle,
$$

where $\bar{M}$ denotes the mean value of the masses of the initial and final nuclei. The closure approximation is not applied to the NME of the $2 v \beta \beta$ decay. $M_{\mathrm{GT}}^{(2 v)}$ of the QRPA is written using the two sets of the intermediate states as

$$
M_{\mathrm{GT}}^{(2 v)}(\mathrm{QRPA})=\sum_{B_{I} B_{F}} \frac{1}{E_{B}-\bar{M}}\left\langle F\left|\sigma \tau^{-}\right| B_{F}\right\rangle \cdot\left\langle B_{F} \mid B_{I}\right\rangle\left\langle B_{I}\left|\sigma \tau^{-}\right| I\right\rangle .
$$

$E_{B}$ is the energy of the intermediate state. We use $E_{B I}$, with an energy calibration, for $E_{B}$ because the QRPA is better for ${ }^{48} \mathrm{Ca}$ than it is for ${ }^{48} \mathrm{Ti}$. The equation for $M_{\mathrm{F}}^{(2 v)}$ can be obtained by removing the spin operators in Eqs. (25) and (26). The intermediate states that contribute to $M_{\mathrm{GT}}^{(2 v)}$ and $M_{\mathrm{F}}^{(2 v)}$ are different. The $2 \nu \beta \beta$ NME is calculated as

$$
M^{(2 v)}=M_{\mathrm{GT}}^{(2 v)}-\left(\frac{g_{V}}{g_{A}}\right)^{2} M_{\mathrm{F}}^{(2 v)} .
$$




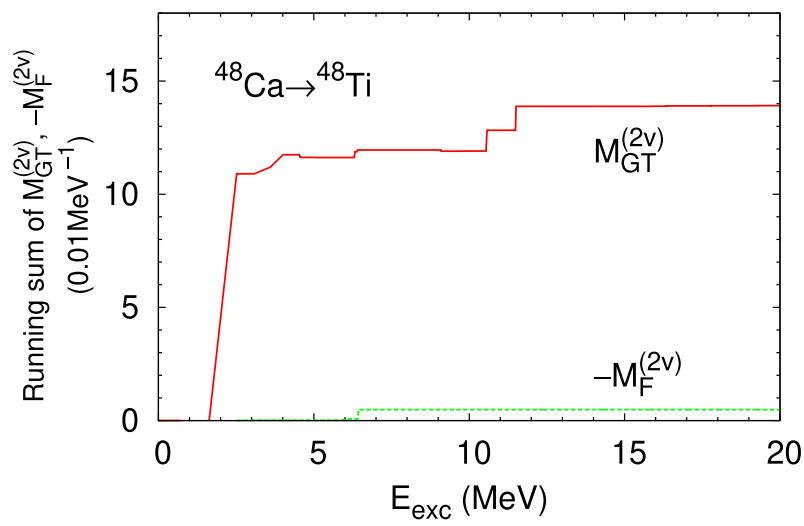

Fig. $4 M_{\mathrm{GT}}^{(2 v)}$ and $M_{\mathrm{F}}^{(2 v)}$ of the QRPA as functions of $E_{\text {exc }}$ of the intermediate nucleus ${ }^{48} \mathrm{Sc}$. $E_{\text {exc }}$ is obtained from the QRPA energy of the calculation based on the initial state with a calibration energy adjusted to the $1^{+}$state of the lowest energy peak of the experimental GT strength function. No quenching factor was used

The running sums of the components of the QRPA are shown in Fig. 4. The horizontal axis indicates the excitation energy of the intermediate state. $M_{\mathrm{GT}}^{(2 v)}$ and $M_{\mathrm{F}}^{(2 v)}$ of the QRPA are $0.139 \mathrm{MeV}^{-1}$ and $-0.0048 \mathrm{MeV}^{-1}$, respectively. $M_{\mathrm{F}}^{(2 v)}$ is very small compared with $M_{\mathrm{GT}}^{(2 v)}$ because of the approximate isospin invariance. The lowest-energy contributions occupy nearly $85 \%$ of $M_{\mathrm{GT}}^{(2 v)}$, and the second largest contribution around $E_{\mathrm{exc}}=11 \mathrm{MeV}$ is the giant resonance of ${ }^{48} \mathrm{Ca} \rightarrow{ }^{48} \mathrm{Sc}$ (see Fig. 2). For $M_{\mathrm{F}}^{(2 v)}$, almost $100 \%$ is occupied by the lowest energy contribution. The running sum of Fig. 4 indicates that a relatively small valence singleparticle space is sufficient for the convergence of $M_{\mathrm{GT}}^{(2 v)}$ and $M_{\mathrm{F}}^{(2 v)} \cdot M^{(2 v)}$ of $0.142 \mathrm{MeV}^{-1}$ is obtained with the bare values of $g_{A}=1.276$ and $g_{V}=1.0$. This is a result with no quenching factor.

The authors of Ref. [30] derived the quenching factors for the GT operator of 0.74 (the $p f$ valence shell) and 0.71 (the $s d p f$ valence shell) from the experimental data of the $\mathrm{GT}^{+}$and $\mathrm{GT}^{-}$strengths. They calculated $M^{(2 v)}$ with these quenching factors and obtained $0.052 \mathrm{MeV}^{-1}$ (the $p f$ valence shell, GXPF1B interaction) and $0.051 \mathrm{MeV}^{-1}$ (the $s d p f$ valence shell, SDPFMU-DB interaction). The similarity of these two values also indicates the sufficiency of the relatively small valence single-particle space. Their results are similar to the early experimental value of $0.046 \pm 0.004 \mathrm{MeV}^{-1}$ [71]. It is noted, however, that the experimental half-life of ${ }^{48} \mathrm{Ca}$ with respect to the $2 \nu \beta \beta$ decay was recently updated [72] and increased in comparison with the previous one. By using the new data and the method used in Ref. [30], a revised experimental $M^{(2 v)}$ of $0.042 \pm 0.004 \mathrm{MeV}^{-1}$ is obtained.

Let us adjust $M^{(2 v)}$ of the QRPA for comparison using the method in Ref. [30]. We apply the quenching factors of 0.74 and 0.71 of the GT operator to the QRPA result; $M_{\mathrm{GT}}^{(2 v)}$ of the QRPA is reduced by factors of 0.55 and 0.50 , respectively. The $M^{(2 v)}$ of the QRPA becomes $0.0745 \mathrm{MeV}^{-1}$ (the average of the results with the different quenching factors), which is $45 \%$ larger than the shell model value. It is stressed that this difference is much smaller than the corresponding difference in $M^{(0 v)}$, which is nearly a factor of two (see Fig. 3). This is because the relatively small energy region is sufficient for the intermediate states, as the neutrino potential is not used.

Next, we introduce our modification method. The modification of $M^{(2 v)}$ of the QRPA is similar to that of $M^{(0 v)}$ but simpler. In the previous discussion on $M_{\mathrm{GT}}^{(0 v)}$, the NME of the 
Table $9 M^{(2 v)}$ of the shell model and the QRPA with the shell model quenching factor multiplied (original) and those modified by our method (modified). The modification method depends on the model (see text)

\begin{tabular}{lll}
\hline & $M^{2 v}$ of shell model $\left(\mathrm{MeV}^{-1}\right)$ & $M^{2 v}$ of $\mathrm{QRPA}\left(\mathrm{MeV}^{-1}\right)$ \\
\hline Original & 0.0515 & 0.0745 \\
Modified & 0.0608 & 0.0666 \\
\hline
\end{tabular}

QRPA was reduced in the low-energy region by $26 \%\left(1-\sqrt{x x^{\prime}}\right)$ by referring to the ratios of the quenching factors of the shell model and QRPA to simulate the mpmh effects. For $M^{(2 v)}$, we refer to the $\mathrm{GT}^{-}$strength of the first $1^{+}$state at $E_{\text {exc }}=2.5173 \mathrm{MeV}$ (experimental value) [73] because this state is exclusively important for the $2 \nu \beta \beta$ decay of ${ }^{48} \mathrm{Ca}$. The ratio of the $\mathrm{GT}^{-}$strength of the shell model to the corresponding value of the QRPA is 0.894 , which is used as an extra quenching factor for the QRPA $M_{\mathrm{GT}}^{(2 v)}$ in our modification method. As $M_{\mathrm{F}}^{(0 v)}$ discussed in Sect. 5, we do not modify $M_{\mathrm{F}}^{(2 v)}$. We also do not consider modifications in the high-energy region. The modified result of $M^{(2 v)}$ of the QRPA is $0.0666 \mathrm{MeV}^{-1}$ (again the average). This value is $29 \%$ larger than the average shell model value of $0.0515 \mathrm{MeV}^{-1}$.

The modification of $M^{(2 v)}$ of the shell model is made because of the giant resonance. In the QRPA, its contribution to $M_{\mathrm{GT}}^{(2 v)}$ is seen around $E_{\mathrm{exc}}=11 \mathrm{MeV}$ (see Fig. 4), which is in the region where the strength function of the shell model for ${ }^{48} \mathrm{Ti} \rightarrow{ }^{48} \mathrm{Sc}$ with the one major valence shell does not have any strength (see Fig. 2). Thus, the shell model calculation of the double- $\beta$ decay does not have contribution of the giant resonance. Its contribution in the QRPA calculation without quenching is $0.0198 \mathrm{MeV}^{-1}$ (see Fig. 4). We add the value with the quenching corrections

$$
0.0198 \cdot 0.525 \cdot 0.894 \mathrm{MeV}^{-1}=0.0093 \mathrm{MeV}^{-1},
$$

to the shell model value of $0.0515 \mathrm{MeV}^{-1}$ and obtain $0.0608 \mathrm{MeV}^{-1}$, which is only $9 \%$ smaller than the modified QRPA value of $0.0666 \mathrm{MeV}^{-1}$. Therefore, consistency between the two models can be obtained using our modification method. The results of this discussion are summarized in Table 9.

If (i) the relevant energy region is low, (ii) the many-body correlations in the initial and final states are small, and (iii) the configuration mixing by the transition operator is small, the two models are approximately consistent. The first condition is fulfilled for $M^{(2 v)}$ because the neutrino potential is not used. The second condition affects the applicability of the QRPA, which depends on the nucleus. The third condition is better satisfied for $M^{(2 v)}$ than for $M^{(0 v)}$ again because the neutrino potential is not used.

\section{Summary}

We have proposed a new method to estimate the NME components of the double- $\beta$ decays from the information already available and demonstrated that similar results can be obtained for the $0 \nu \beta \beta$ NME of ${ }^{48} \mathrm{Ca} \rightarrow{ }^{48} \mathrm{Ti}$ of the shell model and the QRPA with modifications. The extrapolated $M_{\mathrm{GT}}^{(0 v)}$ of the shell model is 1.26 , which is the average of three very close values, and the modified result of the QRPA is expressed in the range of 1.112-1.860. We speculate that the likely value is close to the mean value of 1.486 rather than the edge values. The difference between the two methods was a factor of two before our modifications. 
The current shell model with the one major valence shell is not sufficient for the $0 v \beta \beta$ decay, and the QRPA does not have as many mpmh effects as the shell model has. The result of the shell model was modified by speculating regarding the dependence on the particle-hole energy representing the valence shells based on the intermediate-state energy dependence of the running sum of the QRPA NME. This speculation is justified in two ways. One is the fact that the increasing rate of the $0 \nu \beta \beta$ GT NME with respect to that representative, or intermediate state, energy is the same between the shell model and QRPA in the discussed range. The other is that the NME of the shell model with a very large valence space should include the NME of the QRPA. The experimental data of the charge-change strength functions were used to clarify the reliable energy region of the shell model. The necessary energy region for the $0 \nu \beta \beta$ NME is rather large because of the neutrino potential. The QRPA calculation can be performed up to the convergence of the result with respect to the single-particle as well as intermediate-state energies. Thus, the QRPA was used to modify the shell model result.

The insufficiency of the QRPA was evaluated from the experimental data of the strength function and the shell model calculation in the low-energy region, in which the shell model is very reliable. The mpmh effects seem more important for ${ }^{48} \mathrm{Ti}$ than for ${ }^{48} \mathrm{Ca}$. This analysis was applied to the modification of the QRPA results of the double- $\beta$ NMEs. This application is enabled by the transition density matrix shared by the different phenomena. This modification method for the QRPA has uncertainty in the high-energy region; thus, we considered multiple extreme cases.

The key point of our approach is to combine the information of the different methods in a complementary way, paying attention to the running sums of the NMEs with respect to the intermediate-state energy. In this study, we concentrated on ${ }^{48} \mathrm{Ca} \rightarrow{ }^{48} \mathrm{Ti}$ because sufficient information for our approach is currently available only for this decay instance, i.e., the shell model calculations with different valence single-particle spaces and the data of the strength functions. The GT and Fermi components were investigated separately for the $0 v \beta \beta$ decay. The modified $0 v \beta \beta$ NME was in the middle of the distribution of the NME in many other calculations.

The $2 \nu \beta \beta$ NME was also studied. The NMEs of the shell model and the QRPA were $0.0515 \mathrm{MeV}^{-1}$ and $0.0745 \mathrm{MeV}^{-1}$, respectively. Both values were obtained using the common quenching factors for the GT operators at approximately 0.73 , which is not the fitting parameter for the corresponding experimental value of $0.042 \pm 0.004 \mathrm{MeV}^{-1}$. We obtained a modified shell model result of $0.0608 \mathrm{MeV}^{-1}$ by taking into account the contribution of the giant resonance. We also obtained a modified QRPA result of $0.0666 \mathrm{MeV}^{-1}$ by including an extra quenching factor of 0.894 for the $2 \nu \beta \beta$ NME reflecting the insufficient mpmh effects of the QRPA. If the relevant region of the intermediate-state energy is small, i.e., there is no singularity of the transition operator, and the mpmh correlations are not significant compared with the $1 \mathrm{p} 1 \mathrm{~h}$ correlations, the consistency of the two models can be obtained using simple modifications.

Our approach is important for two reasons. First, the discrepancy problem of the $0 v \beta \beta$ NME using these methods has been unsolved for more than 30 years; it is necessary to clarify the causes. Second, the true values of the $0 \nu \beta \beta$ NME are required regardless of the method used; there is no precondition for which method to use. Thus, we can use as much information as possible. Finally, by extending the wave functions, the improvement in each method, i.e., the shell model, QRPA, and others, should eventually confirm the correct $0 v \beta \beta$ NME by the double convergence mentioned in the introduction.

Acknowledgements This study was supported by the European Regional Development Fund, Project "Engineering applications of microworld physics" (No. CZ.02.1.01/0.0/0.0/16_019/0000766). The numerical cal- 
culations in this study were performed using the computer Oakforest-PACS of the Joint Center for Advanced High Performance Computing through the program of the High Performance Computing Infrastructure in the fiscal year 2020 (hp200001) and the Multidisciplinary Cooperative Research Program 2020 of the Center for Computational Sciences, University of Tsukuba (xg18i006).

Open Access This article is licensed under a Creative Commons Attribution 4.0 International License, which permits use, sharing, adaptation, distribution and reproduction in any medium or format, as long as you give appropriate credit to the original author(s) and the source, provide a link to the Creative Commons licence, and indicate if changes were made. The images or other third party material in this article are included in the article's Creative Commons licence, unless indicated otherwise in a credit line to the material. If material is not included in the article's Creative Commons licence and your intended use is not permitted by statutory regulation or exceeds the permitted use, you will need to obtain permission directly from the copyright holder. To view a copy of this licence, visit http://creativecommons.org/licenses/by/4.0/.

\section{References}

1. W.H. Furry, Phys. Rev. 56, 1184 (1939)

2. Y. Fukuda, T. Hayakawa, E. Ichihara, K. Inoue, K. Ishihara, H. Ishino, Y. Itow, T. Kajita, J. Kameda, S. Kasuga et al., Phys. Rev. Lett. 81, 1562 (1998)

3. Q.R. Ahmad, R.C. Allen, T.C. Andersen, J.D. Anglin, J.C. Barton, E.W. Beier, M. Bercovitch, J. Bigu, S.D. Biller, R.A. Black et al., Phys. Rev. Lett. 89, 011301 (2002)

4. K. Eguchi, S. Enomoto, K. Furuno, J. Goldman, H. Hanada, H. Ikeda, K. Ikeda, K. Inoue, K. Ishihara, W. Itoh et al., Phys. Rev. Lett. 90, 021802 (2003)

5. E. Aliu, S. Andringa, S. Aoki, J. Argyriades, K. Asakura, R. Ashie, H. Berns, H. Bhang, A. Blondel, S. Borghi et al., Phys. Rev. Lett. 94, 081802 (2005)

6. https://wwwkm.phys.sci.osaka-u.ac.jp/en/research/r01.html. (CANDLES project)

7. https://www.mpi-hd.mpg.de/gerda/. (GERDA project)

8. https://www.sanfordlab.org/feature/majorana-demonstrator. (Majorana-Demonstrator project)

9. http://legend-exp.org/. (LEGEND project)

10. https://supernemo.org/. (SuperNEMO project)

11. S. Cebrian, Prog. Part. Nucl. Phys. 114, 103807 (2020). ZICOS project

12. M.H. Lee, J. Inst. 15, C08010 (2020). AMoRE project

13. I.C. Bandac, A.S. Barabash, L. Bergé, M. Brière, C. Bourgeois, P. Carniti, M. Chapellier, M. de Combarieu, I. Dafinei, F.A. Danevich et al., J. High Energy Phys. 2020, 18 (2020). CROSS project

14. https://cuore.lngs.infn.it/en. (CUORE project)

15. https://falcon.phy.queensu.ca/SNO+/. (SNO+ project)

16. A. Gando, Y. Gando, T. Hachiya, A. Hayashi, S. Hayashida, H. Ikeda, K. Inoue, K. Ishidoshiro, Y. Karino, M. Koga et al., Phys. Rev. Lett. 117, 082503 (2016). KamLAND-Zen project

17. https://nexo.llnl.gov/. (nEXO project)

18. https://next.ific.uv.es/next/. (NEXT project)

19. C. Brofferio, O. Cremonesi, S. Dell'Oro, Front. Phys. 7, 86 (2019). https://doi.org/10.3389/fphy.2019. 00086 (CUPID project)

20. https://web.infn.it/lucifer/. (LUCIFER project)

21. www.cobra-experiment.org. (COBRA project)

22. https://darwin.physik.uzh.ch/. (DARWIN project)

23. D.S. Akerib, C.W. Akerlof, A. Alqahtani, S.K. Alsum, T.J. Anderson, N. Angelides, H.M. Araújo, J.E. Armstrong, M. Arthurs, X. Ba et al., Phys. Rev. C 102, 014602 (2020). LUX-ZEPLIN project

24. https://www.katrin.kit.edu/. (KATRIN project)

25. https://www.project8.org/. (Project 8)

26. https://www.kip.uni-heidelberg.de/echo/. (ECHo project)

27. A. Faessler, J. Phys. Conf. Ser. 337, 012065 (2012)

28. J. Engel and J. Menéndez. Rep. Prog. Phys. 80, 046301 (2017)

29. J. Suhonen, From Nucleons to Nucleus: Concepts of Microscopic Nuclear Theory (Springer-Verlag, Berlin, 2007)

30. Y. Iwata, N. Shimizu, T. Otsuka, Y. Utsuno, J. Menéndez, M. Honma, T. Abe, Phys. Rev. Lett. 116, 112502 (2016)

31. C.F. Jiao, J. Engel, J.D. Holt, Phys. Rev. C 96, 054310 (2017)

32. J. Toivanen, J. Suhonen, Phys. Rev. Lett. 75, 410 (1995) 
33. F. Šimkovic, J. Schwieger, G. Pantis, A. Faessler, Found. Phys. 27, 1275 (1997)

34. F. Šimkovic, V. Rodin, A. Faessler, P. Vogel, Phys. Rev. C 87, 045501 (2013)

35. E.D. Commins, P.H. Bucksbaum, Weak Interactions of Leptons and Quarks (Cambridge University Press, Cambridge, 1983)

36. B. Märkisch, H. Mest, H. Saul, X. Wang, H. Abele, D. Dubbers, M. Klopf, A. Petoukhov, C. Roick, T. Soldner, D. Werder, Phys. Rev. Lett. 122, 242501 (2019)

37. I.S. Towner, Phys. Rep. 155, 263 (1987)

38. L. Coraggio, N. Itaco, Front. Phys. 8, 345 (2020). https://doi.org/10.3389/fphy.2020.00345

39. S.J. Novario, P. Gysbers, J. Engel, G. Hagen, G.R. Jansen, T.D. Morris, P. Navrátil, T. Papenbrock, and S. Quaglioni. e-print arXiv:2008.09696 (2020)

40. W.C. Haxton, G.J. Stephenson Jr., Prog. Part. Nucl. Phys. 12, 409 (1984)

41. M. Doi, T. Kotani, E. Takasugi, Prog. Theor. Phys. Suppl. 83, 1 (1985)

42. J. Kotila, F. Iachello, Phys. Rev. C 85, 034316 (2012)

43. M. Horoi, S. Stoica, Phys. Rev. C 81, 024321 (2010)

44. F. Šimkovic, R. Hodák, A. Faessler, P. Vogel, Phys. Rev. C 83, 015502 (2011)

45. J. Terasaki, Phys. Rev. C 91, 034318 (2015)

46. J. Terasaki, Phys. Rev. C 86, 021301(R) (2012)

47. J. Terasaki, Phys. Rev. C 87, 024316 (2013)

48. J. Terasaki, Phys. Rev. C 97, 034304 (2018)

49. J. Menéndez, T.R. Rodríguez, G. Martínez-Pinedo, A. Poves, Phys. Rev. C 90, 024311 (2014)

50. L. Coraggio, A. Gargano, N. Itaco, R. Mancino, F. Nowacki, Phys. Rev. C 101, 044315 (2020)

51. J. Kostensalo, J. Suhonen, Phys. Lett. B 802, 135192 (2020)

52. J.M. Yao, B. Bally, J. Engel, R. Wirth, T.R. Rodríguez, H. Hergert, Phys. Rev. Lett. 124, 232501 (2020)

53. A. Belley, C.G. Payne, S.R. Stroberg, T. Miyagi, J.D. Holt, Phys. Rev. Lett. 126, 042502042502 (2021)

54. J. Suhonen, J. Phys. G 19, 139 (1993)

55. J. Bartel, P. Quentin, M. Brack, C. Guet, H.-B. Håkansson, Nucl. Phys. A 386, 79 (1982)

56. A. Bohr, B.R. Mottelson, Nuclear Structure, Volume I: Single-Particle Motion (Benjamin, New York, 1969)

57. J. Terasaki, Phys. Rev. C 102, 044303 (2020)

58. Y. Iwata, N. Shimizu, Y. Utsuno, M. Honma, T. Abe, T. Otsuka, JPS Conf. Proc. 6, 030057 (2015)

59. K. Yako, M. Sasano, K. Miki, H. Sakai, M. Dozono, D. Frekers, M.B. Greenfield, K. Hatanaka, E. Ihara, M. Kato et al., Phys. Rev. Lett. 103, 012503 (2009)

60. F. Minato, Phys. Rev. C 93, 044319 (2016)

61. N. Schwierz, I. Wiedenhöver, and A. Volya. e-print arXiv:0709.3525 (2007)

62. G.F. Bertsch, I. Hamamoto, Phys. Rev. C 26, 1323 (1982)

63. N.D. Dang, A. Arima, T. Suzuki, S. Yamaji, Phys. Rev. Lett. 79, 1638 (1997)

64. C. Robin, E. Litvinova, Phys. Rev. Lett. 123, 202501 (2019)

65. E. Caurier, J. Menéndez, F. Nowacki, A. Poves, Phys. Rev. Lett. 100, 052503 (2008)

66. M.N. Harakeh, A. van der Woude, Giant Resonances: Fundamental High-Frequency Modes of Nuclear Excitation (Oxford University Press, Oxford, 2001)

67. A.P. Severyukhin, S. Åberg, N.N. Arsenyev, R.G. Nazmitdinov, Phys. Rev. C 98, 044319 (2018)

68. P. Puppe, D. Frekers, T. Adachi, H. Akimune, N. Aoi, B. Bilgier, H. Ejiri, H. Fujita, Y. Fujita, M. Fujiwara et al., Phys. Rev. C 84, 051305(R) (2011)

69. J. Terasaki, J. Engel, G.F. Bertsch, Phys. Rev. C 78, 044311 (2008)

70. K.L.G. Heyde, The Nuclear Shell Model (Springer-Verlag, Berlin, 1990)

71. A.S. Barabash, Nucl. Phys. 935, 52 (2015)

72. A.S. Barabash, in Workshop on Calculation of Double-beta-decay Matrix Elements (MEDEX'19). ed. by O. Civitarese, I. Stekl, J. Suhonen (AIP Publishing, Melville, 2019), p. 020002

73. National Nuclear Data Center, Brookhaven National Laboratory, http://www.nndc.bnl.gov 